\title{
Circular RNA CSNK1G1 promotes the progression of osteoarthritis by targeting the miR-4428/FUT2 axis
}

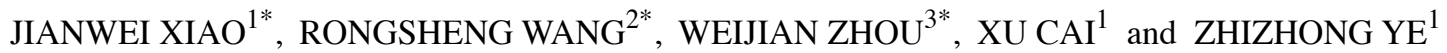 \\ ${ }^{1}$ Shenzhen Futian Hospital for Rheumatic Diseases, Shenzhen, Guangdong 518000; \\ ${ }^{2}$ Shanghai Guanghua Hospital of Integrated Traditional Chinese and Western Medicine, Shanghai 200000; \\ ${ }^{3}$ Yunnan Provincial Hospital of Traditional Chinese Medicine, Kunming, Yunnan 650000, P.R. China
}

Received April 30,2020; Accepted August 12, 2020

DOI: $10.3892 /$ ijmm.2020.4772

\begin{abstract}
Osteoarthritis (OA) is a chronic disease that results in chronic arthralgia and functional disability of the affected joint. To date, there is no effective treatment available for this disease. Circular RNAs (circRNAs) are a type of intracellular stable RNA that can regulate the development and progression of OA. However, the function of circCSNK1G1 in OA has not yet been investigated. In the present study, it was found that circCSNK1G1 was upregulated in OA cartilage tissues. The upregulation of circCSNK1G1 was associated with extracellular matrix (ECM) degradation and chondrocyte apoptosis. Moreover, the expression of miR-4428 was downregulated and that of fucosyltransferase 2 (FUT2) was upregulated in OA-affected cartilage tissues. Dual-luciferase reporter assay and RNA immunoprecipitation confirmed that miR-4428 targeted FUT2 mRNA to inhibit FUT2 expression. circCSNK1G1 and FUT2 induced ECM degradation and chondrocyte apoptosis. The negative effects of circCSNK1G1 and FUT2 were reversed by miR-4428. On the whole, the present study demonstrates that circCSNK1G1 promotes the development of OA by targeting the miR-4428/FUT2 axis. Thus, the circCSNK1G1/miR-4428/FUT2 axis may present a novel target for the treatment of $\mathrm{OA}$ in the clinical setting.
\end{abstract}

\section{Introduction}

Osteoarthritis (OA) is a chronic disease characterized by joint cartilage damage, chronic arthralgia and functional disability; no effective treatment for this disease yet exists in the clinical setting. Patients with OA experience arthralgia, joint stiffness,

Correspondence to: Dr Zhizhong Ye, Shenzhen Futian Hospital for Rheumatic Diseases, 22 Nonglin Road, Futian, Shenzhen, Guangdong 518000, P.R. China

E-mail: ericliang1840@163.com

\section{*Contributed equally}

Key words: circular RNA CSNK1G1, miR-4428, osteoarthritis, chondrocyte, fucosyltransferase 2 periarticular tenderness, arthrocele and limitations in locomotor function. These symptoms reduce the quality of life of affected patients $(1,2)$. Articular cartilage consists of chondrocytes and extracellular matrix (ECM). The ECM is formed from chondrocyte-secreted collagen and proteoglycans. In the process of $\mathrm{OA}$, upregulated levels of matrix metalloproteinases (MMPs) in the cartilage degrade the ECM, and the induced inflammatory responses trigger the apoptosis of chondrocytes, which further decreases the ability of cartilage to secrete ECM components. However, accurate details of the molecular mechanisms of OA remain unknown. Thus, clarifying the mechanisms responsible for the development of OA and identifying key target genes invovled in this process are of utmost importance.

Circular RNAs (circRNAs) are a group of non-coding RNAs of which the $3^{\prime}$ and $5^{\prime}$ ends are connected to form a circular shape by back-splicing $(3,4)$. Due to their shape, circRNAs are resistant to RNase digestion and their expression is more stable in cells compared with linear mRNAs. circRNAs were previously regarded as a splicing by-product (5). Recently, however, circRNAs have been found to be abundant in almost all types of tissues. circRNAs are evolutionarily conserved, are tissue- and development-specific and have been proven to be expressed in OA (6-9). circRNAs function as miRNA sponges that can inhibit the role of miRNAs in restraining targeted expression (10). Previous studies have demonstrated that miRNAs, such as miR-142-5p (11), miR-1202 (12) and miR-140-3p (13) regulate the development of $\mathrm{OA}$ by targeting various genes and pathways. Thus, the circRNA/miRNA axis may regulate the progression of OA and may thus present a novel treatment target.

Fucosyltransferase 2 (FUT2) is a Golgi stack membrane protein that transfers fucose to the terminal galactose on the glycan chains of cell surfaces $(14,15)$. FUT2 can regulate the progression of a number of diseases, including primary sclerosing cholangitis and acute gastroenteritis (16). It can also regulate the development of oral squamous cell carcinoma (17). FUT2 has been found to be upregulated in OA-affected cartilage (18), which indicates that it may play a role in regulating the development of OA.

The present study investigated the role of circCSNK1G1 in vitro and in vivo to clarify the potential mechanisms of circCSNK1G1 in the development of OA. The results of the present study may provide novel therapeutic targets for the treatment of OA. 


\section{Materials and methods}

Patients and sample collection. A total of 10 patients diagnosed with OA and 10 patients diagnosed with knee joint trauma were included in the present study. OA-affected and normal cartilage tissues were collected from January, 2019 to April, 2020. Patients in the OA group had no obvious cause or history of OA. There were 10 patients in the OA group, including 7 males and 3 females. The age ranged from 67 to 82 years, with an average age of $70.15 \pm 7.17$ years. The control group consisted of 10 cases, 8 males and 2 females. The average age was $69.22 \pm 20.07$ years, ranging from 28 to 78 years. There was no statistically significant difference in age between the groups. All patients were fully informed of the experimental aims and procedures and provided written informed consent. The Ethics Committee of Shenzhen Futian Hospital for Rheumatic Disease approved the study.

Animals and grouping. Adult female Sprague-Dawley rats ( $\mathrm{n}=24$; age, 10 weeks) were obtained from Beijing Vital River Laboratory Animal Technology Co.,Ltd. The rats were randomly divided into 4 groups (i.e., the Control, OA, OA + Len-siNC and Len-si-circCSNK1G1) and kept in a cage with a 12/12 $\mathrm{h}$ day/night cycle and stable humidity and temperature. During the surgery, the rats were anesthetized by an intraperitoneal injection of $0.5 \%$ pentobarbital sodium $(50 \mathrm{mg} / \mathrm{kg}$, Sigma-Aldrich; Merck KGaA). Lentivirus ( $1 \times 10^{9}$ PFU, $\left.20 \mu \mathrm{l}\right)$ was injected into the knee joints of the recipient rats 1 week after surgery (20 $\mu \mathrm{l}$ per joint per rat 2 times a week for 4 weeks) $(n=6$ per group). Food and water were provided ad libitum. At 8 weeks after surgery, the rat orbital venous plexus was used for blood collection. The rats were then sacrificed by $\mathrm{CO}_{2}$ inhalation and the knee joints were harvested. For $\mathrm{CO}_{2}$ inhalation, no gas was used to pre-fill the euthanasia chamber. The animals were placed in the chamber and $100 \% \mathrm{CO}_{2}$ was introduced. The filling rate was approximately $10-30 \% \mathrm{CO}_{2}$ per minute of the volume of the euthanasia chamber. As carbon dioxide levels increase, the animals die slowly and painlessly. After $10 \mathrm{~min}$, each animal was separately examined to confirm death. In the case that death could not be confirmed, the animals were also subjected to cervical dislocation. All animal procedures were performed between March and May, 2019 and were approved by the Ethics Committee of Shenzhen Futian Hospital for Rheumatic Disease.

Rat model of $O A$. The rat model of OA was established by medial collateral ligament transection and destabilization of the medial meniscus (DMM). Lentivirus expressing circCSNK1G1 shRNA and the negative control were cloned into pLenti-EF1a-EGFP-F2A-Puro (Biovector Inc.). The lentivirus (1X10 $\left.10^{9} \mathrm{PFU}, 20 \mu \mathrm{l}\right)$ was injected into the knee joints of the rats 1 week post-OA establishment surgery; this treatment was repeated twice a week for 4 weeks. At 2 months after the initial surgery, joint cartilage tissues were collected.

Hematoxylin and eosin $(H \& E)$ staining. The synovial membrane of the right knee joint and cartilage of the tibial plateau were obtained and fixed in $10 \%$ neutral formaldehyde solution. Decalcification in $10 \%$ EDTA was then carried out for 56 days. Conventional dehydration and paraffin embedding were then performed. Specimens were obtained by slicing into sections measuring of $4 \mu \mathrm{m}$ in thickness and routinely dewaxed and washed with xylene. At room temperature, the slices were stained with hematoxylin for $5 \mathrm{~min}$, then $0.5 \%$ eosin solution for 3 min (Beyotime Institute of Biotechnology, Inc.) and then washed with tap water. The slices were then dehydrated in alcohol (from a low to high concentration) and sealed with neutral resin. The morphology of the cartilage tissues was observed under a normal microscope (Nikon Corporation).

Cartilage staining (Safranin $O$ method). Specimens were subjected to $10 \%$ formalin fixation, decalcification and paraffin sectioning (4- $\mu \mathrm{m}$-thick). At room temperature, the specimens were stained with fresh Weigert's stain solution for 3-5 min and differentiated with acid ethanol for $15 \mathrm{sec}$. Subsequently, the specimens were rinsed with distilled water for $10 \mathrm{~min}$. At room temperature, the specimens were stained with solid green dye solution (Beijing Solarbio Science \& Technology Co., Ltd.) for $5 \mathrm{~min}$, and then washed with distilled water for $1 \mathrm{~min}$. Safranin O stain was added to the specimens for 1-2 min and rinsed off with distilled water for $1 \mathrm{~min}$. Specimens were dehydrated with $95 \%$ ethanol and anhydrous ethanol respectively. Finally, the specimens were made transparent with xylene and sealed with optical resin (Ni-E; Nikon Corporation).

TargetScan analysis. TargetScan is a software specifically designed to analyze miRNA target genes in mammals (http://www.targetscan.org/vert_72/). TargetScan supports both Gene Symbol and Emsembl ID formats. In the present study, FUT2 was entered into the he search box. The retrieval results provided multiple transcripts of FUT2 and the corresponding length of the 3 'UTR region. By clicking on each transcript ID, the miRNA binding site on the transcript can be viewed.

Chondrocyte culture. C28/I2 (AC339995, human chondrocytes) cells were purchased from Ze Ye Biotechnology Co., Ltd. and maintained in Dulbecco's modified Eagle's medium (DMEM) with fetal bovine serum (FBS, 10\%). The culture medium was replaced with fresh medium every second day of cell culture. Interleukin (IL)-1 $\beta$ (10 ng/ml, 24 h; ACROBiosystems) was used to induce chondrocyte apoptosis in vitro.

Proliferation assay. C28/I2 cells were cultured in a 96-well plate to detect their proliferative ability. Briefly, $1 \times 10^{4}$ cells were seeded into each well of the plate and cultured in DMEM with 10\% FBS. CCK-8 reagent (100 $\mu \mathrm{l})$ (MedChem Express) was then added to each well. Following incubation for $2 \mathrm{~h}$ in $37^{\circ} \mathrm{C}$, the plate was transferred to an enzyme-linked immunosorbent assay (ELISA) plate reader (Thermo Fisher Scientific, Inc.) to detect the absorbance of the solution at $450 \mathrm{~nm}$.

Flow cytometric assay. The percentage of apoptotic chondrocytes was detected by flow cytometry using an FITC-Annexin V apoptosis detection kit (Beyotime Institute of Biotechnology, Inc.). The cells were then collected and rinsed with PBS, and FITC-Annexin V (300 ng/ml, $\left.4^{\circ} \mathrm{C}, 10 \mathrm{~min}\right)$ was applied to label apoptotic cells. Coupling solution was applied to wash the labeled cells. The cells were then incubated with propidium iodide for $5 \mathrm{~min}$ in $37^{\circ} \mathrm{C}$. A LSRFortessa flow 
cytometer (BD Biosciences) was used to obtain the required data, and FlowJo software version 7.6.1. was applied to analyze the data.

Western blot analysis. The expression of cleaved caspase-3, cleaved caspase-9, Bax and Bcl-2 in the cultured chondrocytes was detected by western blot analysis. The cultured C28/I2 cells were harvested and lysed with RIPA buffer (Beyotime Institute of Biotechnology, Inc.). The protein concentration of the samples was then determined using a BCA kit (Beyotime Institute of Biotechnology, Inc.). The proteins were then separated by $10 \%$ SDS-PAGE and transferred to a PVDF membrane. The membrane was blocked with $5 \%$ non-fat milk, and the proteins were probed with the necessary primary antibodies [cleaved caspase-3 (ab2302, 1:1,000; AbcamA), cleaved caspase-9 (ab2324, 1:1,000; Abcam), Bax (ab3191, 1:1,000; Abcam), Bcl-2 (ab117115, 1:2,000; Abcam)] and a secondary antibody [goat anti-rabbit IgG-HRP (ab97051, 1:1,000; Abcam)]. The membranes were then subjected to luminescence with an ECL kit (P0018FS; Beyotime Institute of Biotechnology, Inc.) and exposure with the Chemiluminescent gel imaging system (Tanon-5200; Tanon Science \& Technology Co., Ltd.). Images were collected for analysis.

$R T-q P C R$. The expression of circCSNK1G1, miR-4428, MMP-13, collagen II, aggrecan, FUT2 and cytochrome $c$ (Cyt-c) in human and rat cartilage and cultured chondrocytes was detected by RT-qPCR as previously described (19). Briefly, tissues and cells were harvested and lysed with TRIzol reagent (Invitrogen; Thermo Fisher Scientific, Inc.). RNA concentrations were then determined using a NanoDrop 2000 instrument. cDNA for circCSNK1G1, MMP-13, collagen II, aggrecan, FUT2 and Cyt-c was synthesized with PrimeScript RT Master Mix (Takara Bio, Inc.), and cDNA for miRNA was synthesized with a RevertAid First Strand cDNA Synthesis kit (Thermo Fisher Scientific, Inc.). RT-qPCR was conducted using the miScript SYBR ${ }^{\circledR}$-Green PCR kit (Thermo Fisher Scientific, Inc.). All detections were conducted at least 3 times, and the primers used are listed in Table I. The PCR thermal cycling parameters were as follows: PCR thermal cycling parameters: $95^{\circ} \mathrm{C}$ for $5 \mathrm{~min}$, followed by the 3 -step reaction: Denaturation at $94^{\circ} \mathrm{C}$ for $30 \mathrm{sec}$, annealing at $60^{\circ} \mathrm{C}$ for $30 \mathrm{sec}$, and 45 cycles. The results were analyzed using the $2^{-\Delta \Delta \mathrm{Cq}}$ method (19).

Enzyme-linked immunosorbent assay. The expression of IL-6, tumor necrosis factor (TNF)- $\alpha$ and lactate dehydrogenase (LDH) in rat serum was detected by ELISA using respective kits (R\&D Systems, Inc.) in accordance with the manufacturer's instructions.

Terminal deoxynucleotidyl transferase dUTP nick-end labeling (TUNEL) staining. Apoptotic cells in rat cartilage were labeled using a TUNEL assay kit (Beyotime Institute of Biotechnology, Inc.) according to the manufacturer's instructions. TUNEL-positive cell numbers were calculated manually with the help of a microscope (Nikon). Apoptotic cell numbers were counted from 10 random fields per slide.

Cell transfection. si-circCSNK1G1 (GCAATCAAACTG GAACCAA), miR-4428 mimics, miR-NC, siNC (TTACAT
GGCAACCTACCTT), AMO-4428 and siFUT2 (CCTGTA ATGCTCGCACTTT) were designed and synthesized by GenPharma. Lipofectamine 2000 (Life Technologies) was used for transfection, and the transfection procedure was according to the instructions of Lipofectamine 2000. The concentrations used for transfection were as follows: siNC $(50 \mathrm{nM})$, si-circCSNK1G1 (50 nM), siFUT2 (50 nM), miR-4428 mimics (45 nM), miR-NC (45 nM), AMO-4428 (40 nM). The culture medium was replaced with normal culture medium after $48 \mathrm{~h}$, and culture was continued for a further 2 days.

Dual-luciferase reporter assay. The mRNA 3'UTR of FUT2 was amplified and sub-cloned into the pMIR-GLO reporter plasmid (Promega Corporation), and the resulting plasmids were named FUT2-WT and FUT2-MUT. Chondrocytes were cultured in a 96-well plate and co-transfected with miR-4428 mimics/NC and FUT2-WT/FUT2-MUT using Lipofectamine 2000 (Life Technologies; Thermo Fisher Scientific, Inc.). After $48 \mathrm{~h}$, luciferase activity was detected using a dual-luciferase reporter assay system (Promega Corporation). The $20 \mu 1$ cell lysate was added to the luminescent plate. Background values were read with a GloMax bioluminescence detector (Promega Corporation) and $100 \mu \mathrm{l}$ LARII solution (Promega Corporation) were added to each sample. After reading, add $100 \mu \mathrm{l}$ Stop\&GloR Reagent (Promega Corporation) was added to each sample. Normalization was carried out according to $\Delta$ activity multiple $=(F / R)$ sample (/F/R) for comparison (F, Firefly luciferase; R, Renilla luciferase).

Statistical analysis. The data are presented as the means \pm SD. GraphPad Prism 6.0 (GraphPad Software, Inc.) was applied for statistical analysis. Differences between 2 groups were analyzed using a Student's t-test, and differences between 2 groups were calculated using one-way analysis of variance (ANOVA) followed by Tukey's multiple comparison test. Pearson's correlation coefficient was used for correlation analysis. A P-value $<0.05$ was considered to indicate a statistically significant difference.

\section{Results}

circCSNK1G1 is upregulated in OA-affected cartilage tissues. To clarify the role of circRNAs in OA, circCSNK1G1 expression was detected in the cartilage tissues of patients with OA. circCSNK1G1 expression was evidently upregulated in OA-affected cartilage compared with normal cartilage tissue $(\mathrm{P}<0.05$; Fig. 1A). These results indicate that circCSNK1G1 may play a regulatory role in the progression of OA. In addition, the expression of circCSNK1G1 was markedly increased in the cartilage tissues of rats with OA compared with the tissue from the control group $(\mathrm{P}<0.05$; Fig. 1B). These results indicate that the upregulation of circCSNK1G1 in OA-affected tissues may play a role in the pathophysiological processes of OA. However, the exact details of this regulatory mechanism require further investigation.

circCSNK1G1 knockdown inhibits inflammation in OA. Lentivirus was applied by an intra-articular injection to knock down the expression of circCSNK1G1 and observe 
Table I. Primer sequences used for RT-qPCR.

\begin{tabular}{lll}
\hline Genes & \multicolumn{1}{c}{ Sense (5'-3') } & \multicolumn{1}{c}{ Antisense (5'-3') } \\
\hline circCSNK1G1 & GCCATCACAACAGCAGCCT & AGGTCAAACAAGTCCTCCAAG \\
miR-4428 & CAAGGAGACGGGAACATGGA & GAACATGTCTGCGTATCTC \\
MMP-13 & CCTTGATGCCATTACCAGTCTCC & AAACAGCTCCGCATCAACCTGC \\
Aggrecan & ACTCTGGGTTTTCGTGACTCT & ACACTCAGCGAGTTGTCATGG \\
FUT2 & CTACCACCTGAACGACTGGATG & AGGGTGAACTCCTGGAGGATCT \\
Cyt-c & AAGGGAGGCAAGCACAAGACTG & CTCCATCAGTGTATCCTCTCCC \\
Collagen II & CCTGGCAAAGATGGTGAGACAG & CCTGGTTTTCCACCTTCACCTG \\
U6 & AGTAAGCCCTTGCTGTCAGTG & CCTGGGTCTGATAATGCTGGG \\
GAPDH & AATGGACAACTGGTCGTGGAC & CCCTCCAGGGGATCTGTTTG
\end{tabular}

Cyt-c, cytochrome $c$; MMP-13, matrix metalloproteinase 13; FUT2, fucosyltransferase 2.

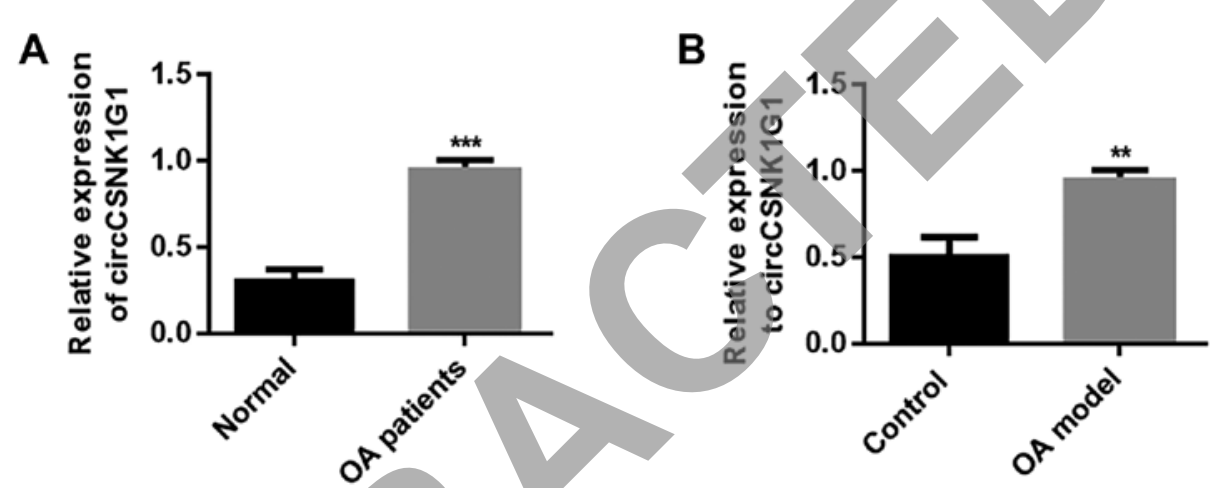

Figure 1. circCSNK1G1 is upregulated in OA-affected cartilage. (A) Expression of circCSNK1G1 in cartilage tissue from patients with OA and normal human cartilage. (B) Expression of circCSNK1G1 in rat cartilage. Data are shown as the means $\pm \mathrm{SD}\left(\mathrm{n}=4\right.$, Student's t-test, $\left.{ }^{* *} \mathrm{P}<0.01,{ }^{* * * *} \mathrm{P}<0.001\right)$. OA, osteoarthritis.

the mechanisms of circCSNK1G1 in OA-affected cartilage. The expression of circCSNK1G1 was first verified. circCSNK1G1 was found to be upregulated in the rat models of OA compared with the control group. However, the application of lentivirus effectively decreased the expression of circCSNK1G1 (Fig. 2A). The expression of IL-6, TNF- $\alpha$ and LDH in serum was then evaluated. The levels of IL-6, TNF- $\alpha$ and LDH were evidently increased in the OA and Len-siNC groups compared with those in the control group (Fig. 2B-D). In addition, the expression of IL- 6 , TNF- $\alpha$ and LDH in articular cartilage was evaluated by RT-qPCR, and the levels of IL-6, TNF- $\alpha$ and LDH were evidently increased in the $\mathrm{OA}$ and Len-siNC groups compared with those in the control group (Fig. 2E-G). However, the levels of IL-6, TNF- $\alpha$ and LDH were decreased by treatment with Len-si-circCSNK1G1 compared with those in the OA and Len-siNC groups, although the levels remained higher than those in the control group.

Chondrocyte apoptosis was evaluated by detecting the expression of cleaved caspase- 3 and cleaved caspase- 9 , and the number of TUNEL-positive cells in cartilage tissues from rats with OA to examine the role of Len-si-circCSNK1G1 in OA. The expression of cleaved caspase- 3 and cleaved caspase- 9 was increased in the OA group compared with the control and Len-siRNA groups. The application of Len-si-circCSNK1G1 markedly decreased the expression of cleaved caspase- 3 and cleaved caspase- 9 compared with the OA and Len-siNC groups (Fig. 2H). In addition, the TUNEL-positive cell numbers were higher in the OA and Len-siNC groups compared with the control group. The application of Len-si-circCSNK1G1 decreased the TUNEL-positive cell numbers compared with the OA and Len-siNC groups (Fig. 2I). H\&E staining was then used to evaluate the integrity of cartilage tissue. In the control group, the surface layer of the cartilage tissue was smooth and flat, the chondrocytes were arranged in an orderly manner, the hierarchical structure was clear, the staining distribution was uniform, the tidal line was complete and no obvious cell clustering could be found. By contrast, in the OA model group, the articular cartilage surface was thinner, local fissure defects were noted, the chondrocytes exhibited a disordered arrangement and the hierarchical structure could not easily be recognized. In the circCSNK1G1 knockdown group, the cartilage surface was slightly roughened, but no obvious cracks were observed. In addition, the chondrocytes were arranged regularly (Fig. 2J). Morphological changes in articular cartilage were also evaluated by saffron solid green staining. In the model group, cartilage degeneration was reduced from compensatory hyperplasia to chondrocytes and stroma; in addition, the cartilage was thin and exhibited signs of exfoliation. The saffron Red O solid green staining in the pericellular matrix became lighter. The knockdown of circCSNK1G1 protected the chondrocytes from these changes (Fig. 2K). On the whole, these results indicate that blocking circCSNK1G1 
A

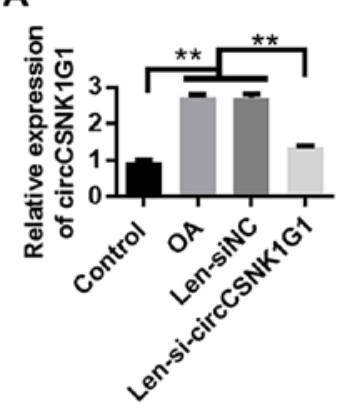

B

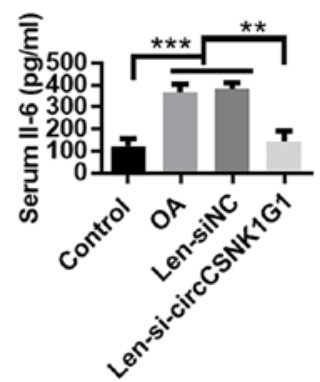

C

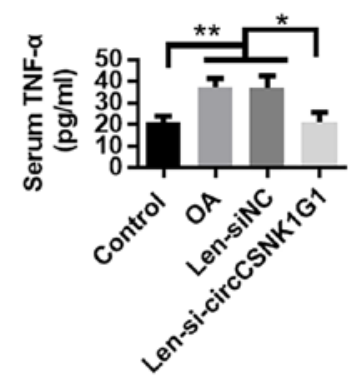

D

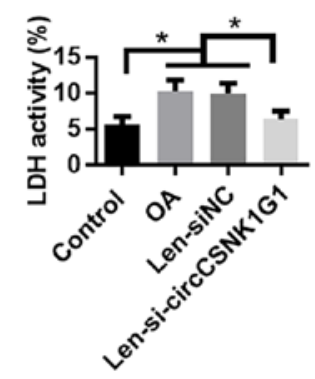

E

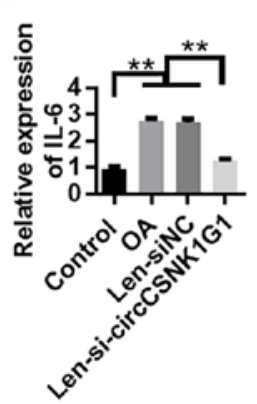

I
$\mathbf{F}$
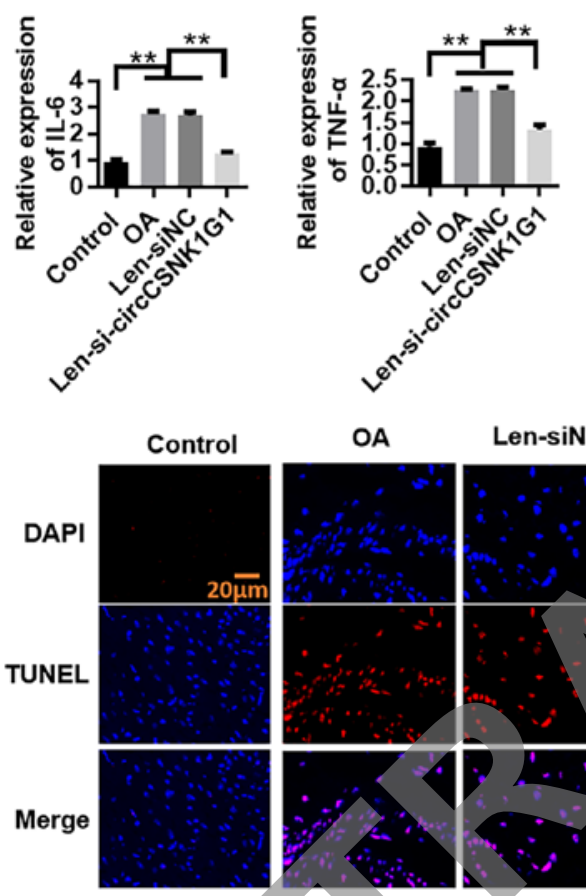

$$
\mathbf{J}
$$
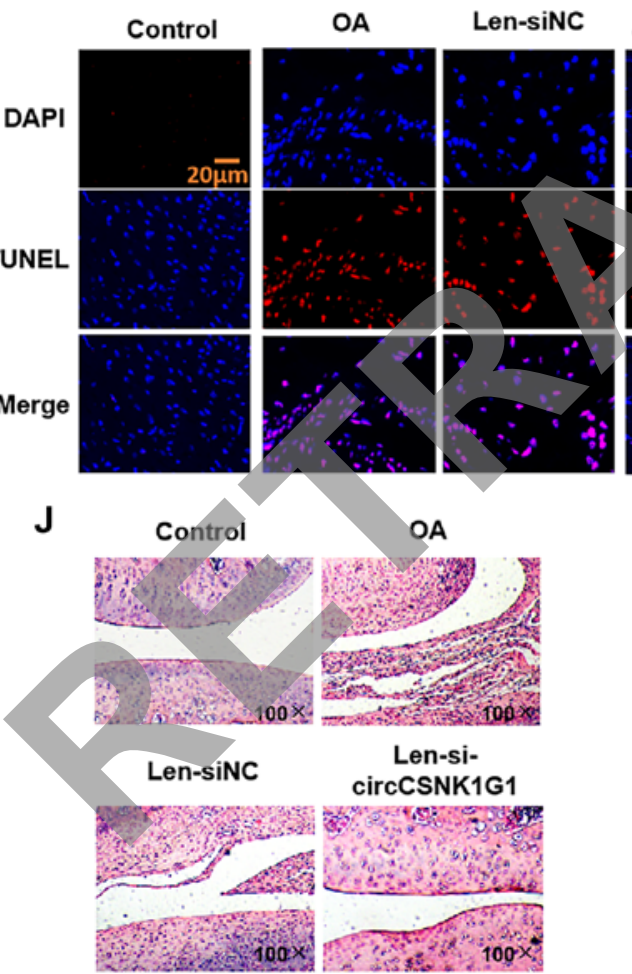

G

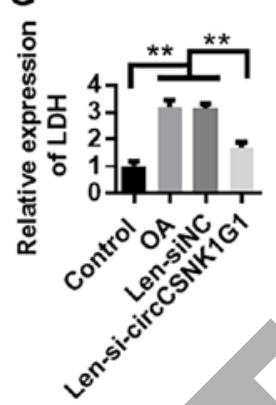

H

Len-si-
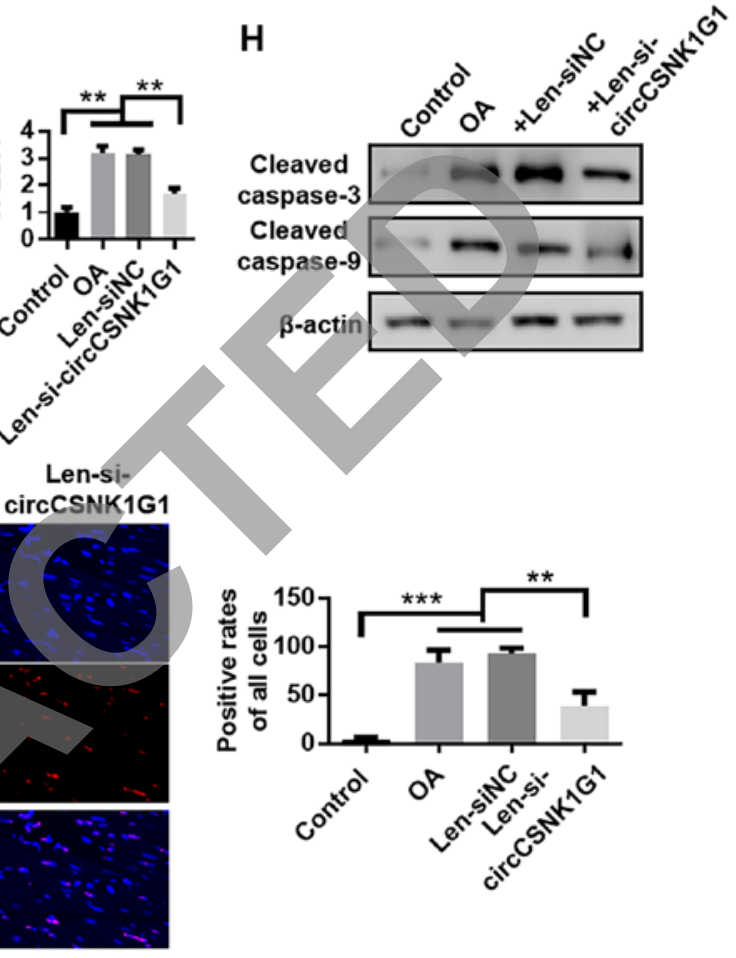

K
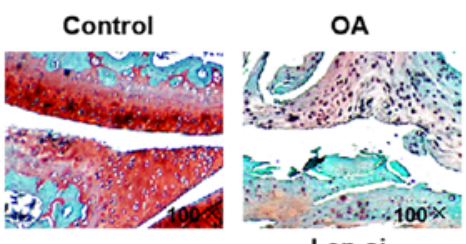

Len-siNC

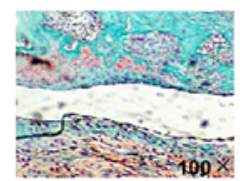

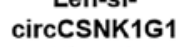
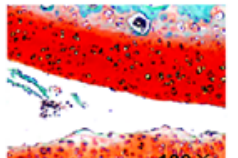

Figure 2. circCSNK1G1 knockdown inhibits inflammation in OA. (A) circCSNK1G1 expression detected by RT-qPCR. (B-D) IL-6, TNF- $\alpha$ and LDH expression in rat serum detected by ELISA. (E-G) IL-6, TNF- $\alpha$ and LDH expression in rat articular cartilage detected by RT-qPCR assay. (H) Cleaved caspase-3 and cleaved-caspase-9 expression in rat cartilage were detected by western blot analysis. (I) TUNEL staining of rat cartilage. (J) Evaluation of cartilage morphology by hematoxylin and eosin staining. (K) Morphological changes in articular cartilage evaluated by saffron solid green staining. Data are shown as the means $\pm \mathrm{SD}\left(\mathrm{n}=4\right.$, ANOVA followed by Tukey's multiple comparison test, $\left.{ }^{*} \mathrm{P}<0.05,{ }^{* *} \mathrm{P}<0.01,{ }^{* * *} \mathrm{P}<0.001\right)$. OA, osteoarthritis; IL-6, interleukin-6; TNF- $\alpha$, tumor necrosis factor- $\alpha$; LDH, lactate dehydrogenase.

expression with Len-si-circCSNK1G1 reduces the expression of inflammatory factors and chondrocyte apoptosis.

circCSNK1G1 promotes chondrocyte apoptosis by sponging $m i R-4428$. Possible interacting miRNAs were predicted by the StarBase (http://starbase.sysu.edu.cn/) database to clarify the mechanisms of circCSNK1G1 in OA. The potential binding sites of miR-4428 on circCSNK1G1 are illustrated in Fig. 3A. The expression of circCSNK1G1 was knocked down by transfection with si-circCSNK1G1 (Fig. S1). The changes in miR-4428 expression in the rat model OA following circCSNK1G1 knockdown by lentivirus were also analyzed (Fig. 3B). The expression of miR-4428 was upregulated following circCSNK1G1 knockdown in the rat 
A

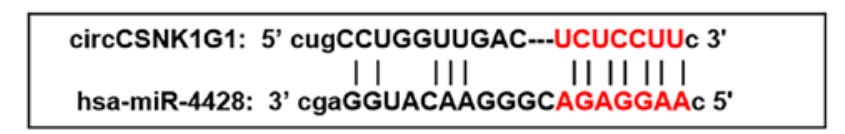

C

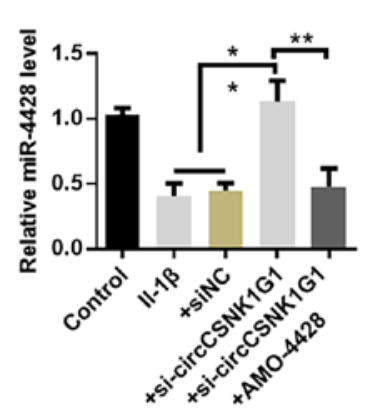

D

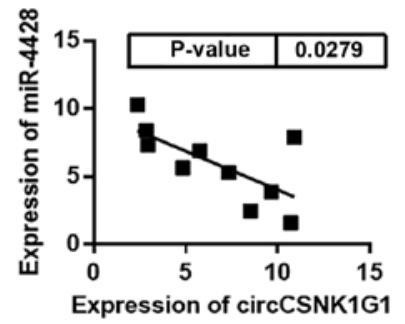

B :

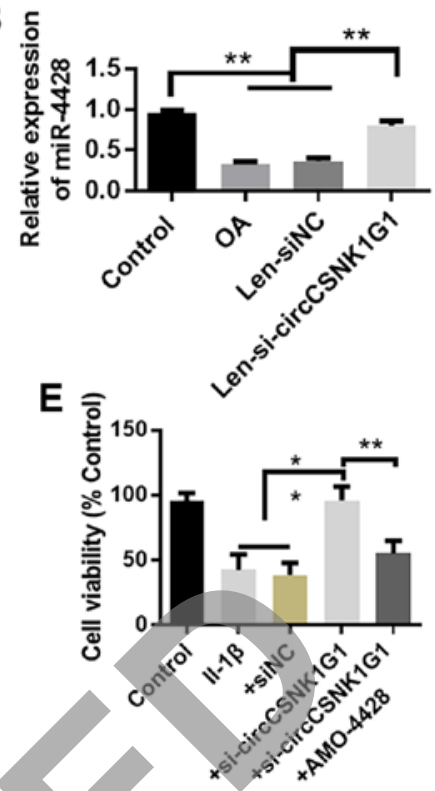

F

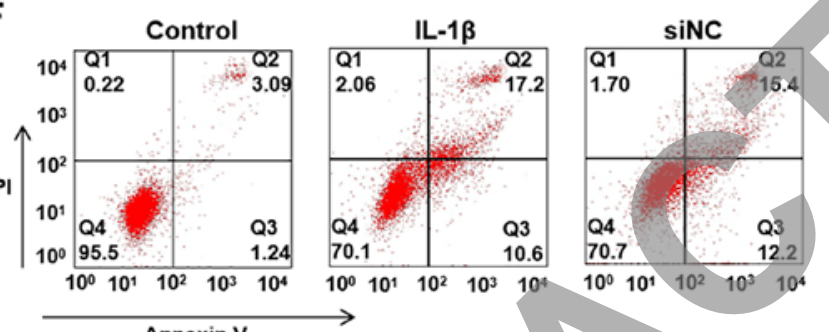

si-circCSNK1G1
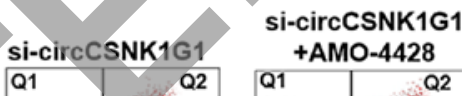

+ AMO-4428

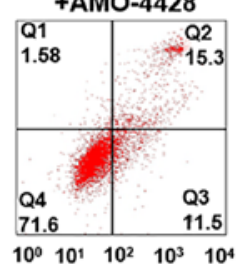

G
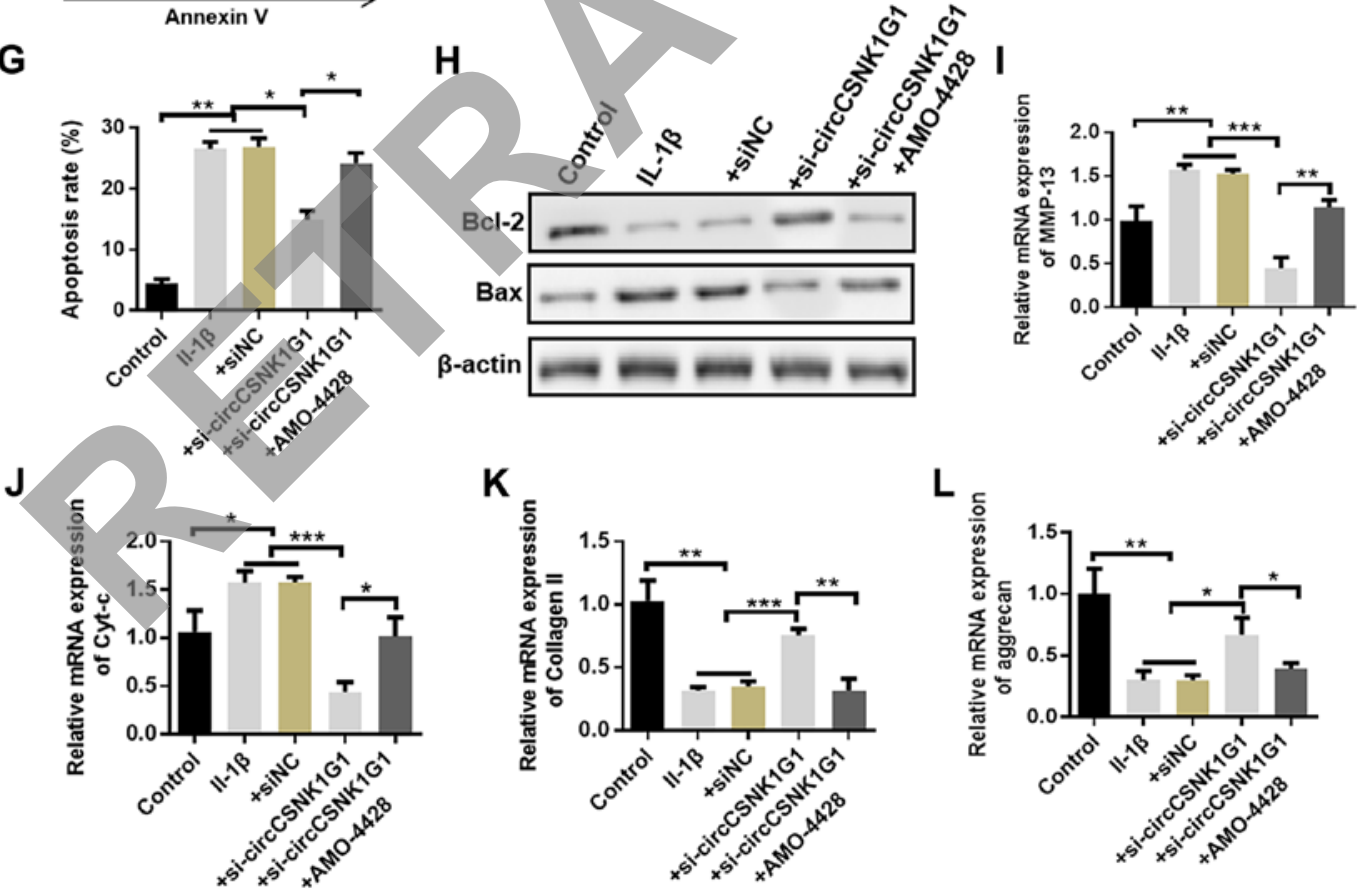

K

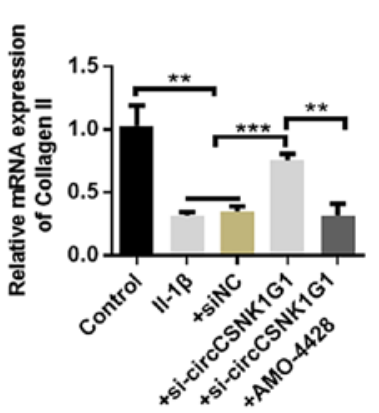

L

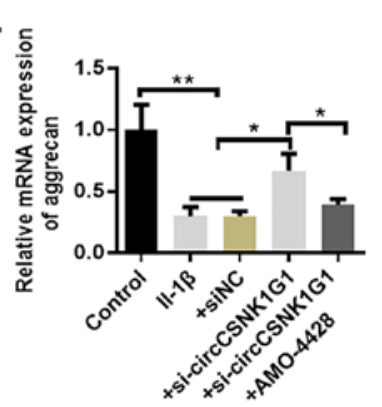

Figure 3. circCSNK1G1 promotes chondrocyte apoptosis by sponging miR-4428. (A) Predicted binding sites of miR-4428 in circCSNK1G1. (B) Detection of the expression of miR-4428 in the rat osteoarthritis (OA) model by RT-qPCR ( $=4$, ANOVA followed by Tukey's multiple comparison test, $\left.{ }^{* *} \mathrm{P}<0.01\right)$. (C) Expression of miR-4428 in cultured chondrocytes detected by RT-qPCR ( $\mathrm{n}=4$, ANOVA followed by Tukey's multiple comparison test, $\left.{ }^{* *} \mathrm{P}<0.01\right)$. (D) Correlation analysis between miR-4428 and circCSNK1G1 expression in cartilage tissue from patients with $\mathrm{OA}(\mathrm{n}=10, \mathrm{r}=-0.6621, \mathrm{P}<0.05)$. (E) Proliferation assays of cultured chondrocytes. (F and G) Percentage of apoptotic cells detected by flow cytometry. (H-L) Expression of Bax, Bcl-2, Cyt-c, MMP-13, collagen II and aggrecan in cultured chondrocytes was detected by western blot analysis or RT-qPCR (ANOVA followed by Tukey's multiple comparison test, "P $<0.05$, $\left.{ }^{* *} \mathrm{P}<0.01,{ }^{* * *} \mathrm{P}<0.001\right)$. Cyt-c, cytochrome $c$; MMP-13, matrix metalloproteinase 13.

joint tissues. The expression of miR-4428 was also detected following transfection with si-circCSNK1G1 in the cultured chondrocytes and it was found to be evidently increased in the si-circCSNK1G1 group compared with the IL-1 $\beta$ and siNC groups (Fig. 3C). In addition, the expression of circCSNK1G1 and miR-4428 was detected in the cartilage tissues of patients 
with OA, and a negative correlation between miR-4428 and circCSNK1G1 expression was observed by Pearson's correlation analysis (Fig. 3D). These results indicate that miR-4428 can be inhibited by circCSNK1G1 in OA.

To demonstrate the role of miR-4428 in OA,AMO- 4428 was transfected into cultured chondrocytes to suppress miR-4428 expression (Fig. S2). IL-1 $\beta$ evidently decreased chondrocyte cell viability compared with the control group; by contrast, transfection with si-circCSNK1G1 evidently increased cell viability compared with the IL- $1 \beta$ group. However, the promoting effect of si-circCSNK1G1 on cell viability was suppressed by transfection with AMO-4428 (Fig. 3E). The percentage of apoptotic cells was also detected by flow cytometry. IL-1 $\beta$ increased the percentage of apoptotic cells, whereas si-circCSNK1G1 decreased this percentage. However, transfection with AMO-4428 attenuated the protective effects of si-circCSNK1G1 compared with the si-circCSNK1G1 group (Fig. 3F and G). In addition, the expression of several apoptosis-related proteins (e.g., Bax and Bcl-2) was detected by western blot analysis, and the mRNA levels of MMP-13, Cyt-c, collagen II and aggrecan were detected by RT-qPCR. The expression of Bax and Cyt-c was upregulated, whereas the expression of $\mathrm{Bcl}-2$ was downregulated in the si-circCSNK1G1 + AMO-4428 group compared with the si-circCSNK1G1 group. The expression of MMP-13 was upregulated, and the expression of collagen II and aggrecan was downregulated in the si-circCSNK1G1 + AMO-4428 group compared with the si-circCSNK1G1 group (Fig. 3H-L). These results indicate that circCSNK1G1 promotes chondrocyte apoptosis by sponging miR-4428.

miR-4428 protects chondrocytes in OA. miR-4428 expression was evidently decreased in the OA group compared with that in the control group (Fig. 4A). In cultured chondrocytes, IL-1 $\beta$ treatment induced a decrease in miR-4428 expression. Moreover, miR-4428 expression was upregulated by transfection with miR-4428 mimic and was downregulated by transfection with AMO-4428 (Figs. 4B, S2 and S3). Cell viability was also evidently repressed in the IL-1 $\beta$ group compared with that in the control group. Transfection with miR-4428 mimic evidently promoted cell viability compared with the IL- $1 \beta$ group. The promoting effect of miR- 4428 mimics was inhibited by transfection with AMO-4428 (Fig. 4C). Flow cytometry also demonstrated that the percentage of apoptotic cells was increased in the IL-1 $\beta$ group. However, transfection with miR-4428 mimics evidently inhibited this effect. The inhibitory effect of miR-4428 mimics on apoptosis was attenuated by AMO-4428 (Fig. 4D and E). Furthermore, the expression of MMP-13, Bax and Cyt-c was increased in the IL-1 $\beta$ and miR-4428 mimics + AMO-4428 groups compared with that in the control and miR-4428 mimics groups. The expression of MMP-13, Bax and Cyt-c was decreased in the miR-4428 mimics group compared with that in the IL-1 $\beta$ and miR-4428 mimics + AMO-4428 groups. The expression of collagen II, aggrecan and Bcl-2 was decreased in the IL-1 $\beta$ and miR-4428 mimics + AMO-4428 groups compared with that in the control and miR-4428 mimics groups. Finally, the expression of collagen II, aggrecan and Bcl-2 was increased in the miR-4428 mimics group compared with that in the IL-1 $\beta$ and miR-4428 mimics + AMO-4428 groups (Fig. 4F-J). These results indicate that miR- 4428 can protect chondrocytes from damage induced by OA.

miR-4428 targets FUT2 in OA. The potential target gene was predicted with TargetScan (http://www.targetscan. org/vert_72/). FUT2 was predicted to be a target gene of miR-4428. The binding site between miR-4428 and FUT mRNA is shown in Fig. 5A. The changes in FUT2 expression following circCSNK1G1 knockdown in the rat model of OA were analyzed and it was found that the expression of FUT2 decreased following circCSNK1G1 knockdown (Fig. 5B). The luciferase activity evidently decreased in the miR-4428 + WT group compared with that in the other 3 groups (Fig. 5C). The expression of FUT2 in the AMO-4428 group was evidently increased compared with that in the control group. Transfection with miR-4428 mimics markedly decreased FUT2 expression (Fig. 5D). The expression of miR-4428 and FUT2 was detected, and a negative correlation between miR-4428 and FUT2 was observed (Fig. 5E).

miR-4428 inhibits chondrocyte apoptosis by targeting FUT2. The expression of FUT2 was evidently promoted by IL-1 $\beta$ in cultured chondrocytes compared with the control group. The application of miR-4428 mimics inhibited FUT2 expression promoted by IL-1 $\beta$ (Fig. 6A). Subsequently, FUT2 siRNA was used to knock down FUT2 expression in cultured chondrocytes. Compared with the control and siNC groups, the expression of FUT2 was evidently decreased in the siFUT2 group (Fig. 6B). The present study then determined whether FUT2 knockdown can protect chondrocytes from the effects of IL-1 $\beta$. Cell viability inhibited by IL- $1 \beta$ was promoted by FUT2 siRNA (Fig. $6 \mathrm{C}, \mathrm{P}<0.01$ ); the increased percentage of apoptotic cells induced by IL-1 $\beta$ was also decreased by FUT2 siRNA (Fig. 6D-E). In addition, the expression of Bax, Cyt-c and MMP-13 was evidently inhibited by FUT 2 siRNA compared with the IL- $1 \beta$ group. Moreover, the expression of Bcl-2 was evidently increased following transfection with FUT2 siRNA compared with that in the IL-1 $\beta$ group (Fig. $6 \mathrm{~F}-\mathrm{J}$ ). These results indicate that FUT2 is a critical gene involved in the development of OA targeted by miR- 4428 .

\section{Discussion}

$\mathrm{OA}$ is a chronic degenerative articular disease characterized by chronic arthralgia, stiffness, declining locomotor ability, and eventually, disability (20). Accurate details of the pathophysiological mechanisms of the disease remain unknown. A previous study indicated that degenerated articular cartilage is the initial change in the development of OA (21). Subsequently, the complex molecular mechanisms of OA have been observed, including the formation of osteophytes, the remodeling of subchondral bone, the abnormal remodeling of cartilage, changes in the synovium, bone marrow lesion, chondrocyte apoptosis and ECM degradation (22-26). Non-coding RNAs, including miRNAs and circRNAs, were recently confirmed to play a regulatory role in the onset and progression of OA. miRNAs are a group of short non-coding RNAs measuring $\sim 22$ nucleotides in length (27). Several miRNAs have been proven to regulate various signaling 
A

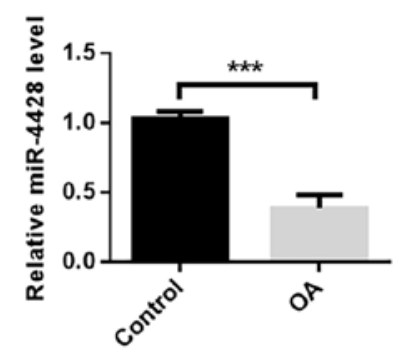

B

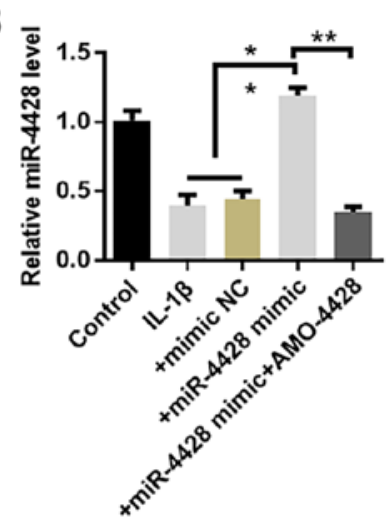

C

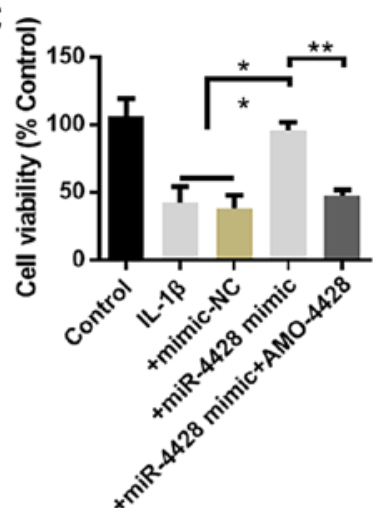

+miR-4428 mimic +AMO-4428

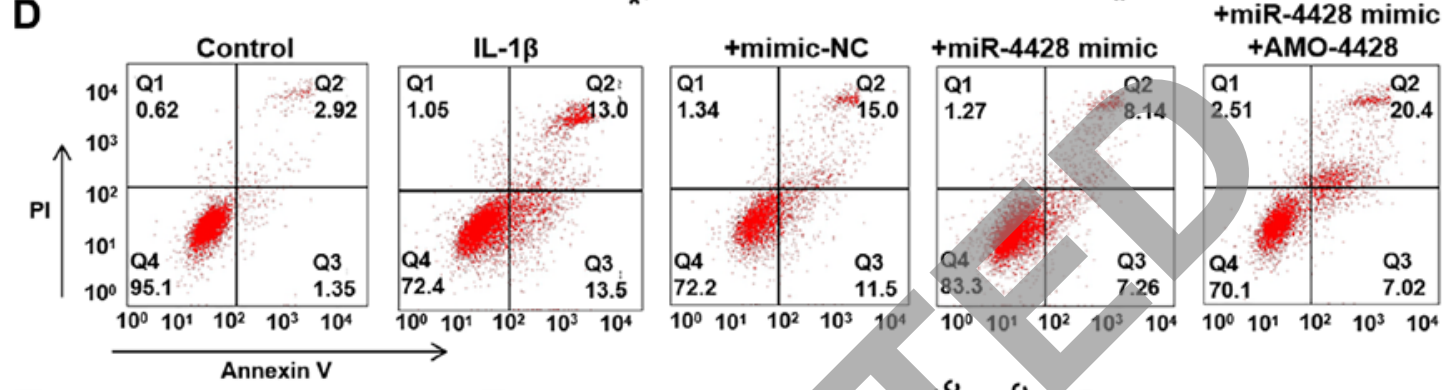

E

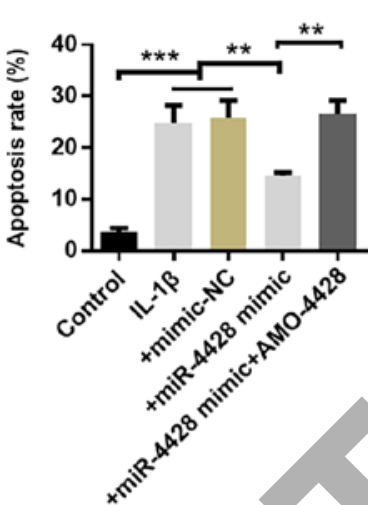

$\mathbf{F}$
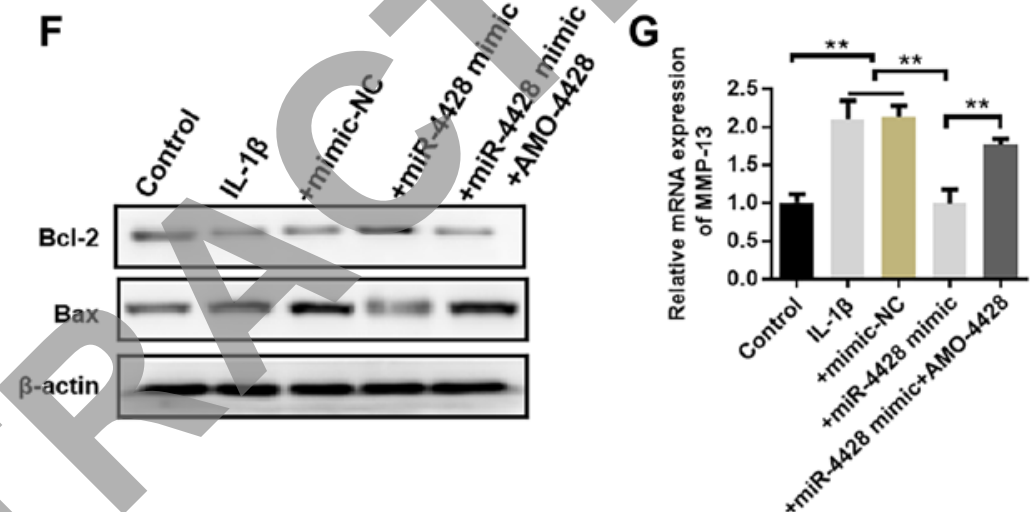

H

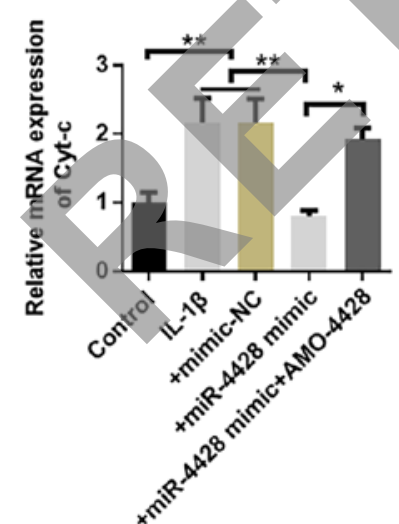

I

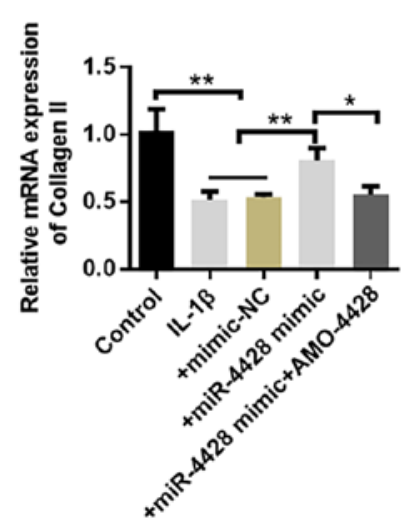

J

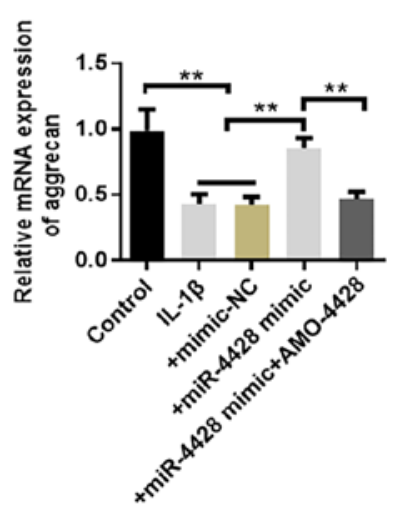

Figure 4. miR-4428 protects chondrocytes in OA. (A) miR-4428 expression in rat cartilage detected by RT-qPCR. (B) Expression of miR-4428 in cultured chondrocytes detected by RT-qPCR. (C) Proliferation assays of cultured chondrocytes. (D and E) Percentage of apoptotic cells detected by flow cytometry. (F-J) Expression of Bax, Bcl-2, Cyt-c, MMP-13, collagen II and aggrecan in cultured chondrocytes was detected by western blot analysis or RT-qPCR ( $\mathrm{n}=4$, ANOVA followed by Tukey's multiple comparison test, $\left.{ }^{*} \mathrm{P}<0.05,{ }^{* *} \mathrm{P}<0.01,{ }^{* * * *} \mathrm{P}<0.001\right)$. OA, osteoarthritis; Cyt-c, cytochrome $c$; MMP-13, matrix metalloproteinase 13.

pathways, apoptosis and chondrocyte functions in OA (28). Some miRNAs are differentially expressed during the onset and development of OA (29-31). A total of 42 circRNAs have also been found to be differentially expressed in OA (32). These circRNAs regulate chondrocyte apoptosis by sponging targeted miRNAs. In the present study, the ceRNA mechanism of the circCSNK1G1/miR-4428/FUT2 that may exist in arthritis was first constructed by referencing and applying the TargetScan (http://www.targetscan.org/vert_72/) and Starbase (http://starbase.sysu.edu.cn/index.php) websites. Furthermore, the role of circCSNK1G1 in aggravating inflammation at the chondrocyte level we confirmed through relevant experiments. Therefore, the function and regulatory mechanisms of circCSNK1G1 were further invesigated in 
A

\begin{tabular}{|lc|}
\hline FUT2 WT: & 5' UUCUUUGAGACCUUUUCUCCUUC 3' \\
I I I I I I | \\
hsa-miR-4428: & 3' CGAGGUACAAGGGCAGAGGAAC 5' \\
I I I I | | | \\
FUT2 MT : & 5' UUCUUUGAGACCUUUAGAGGAC 3' \\
\hline
\end{tabular}

C
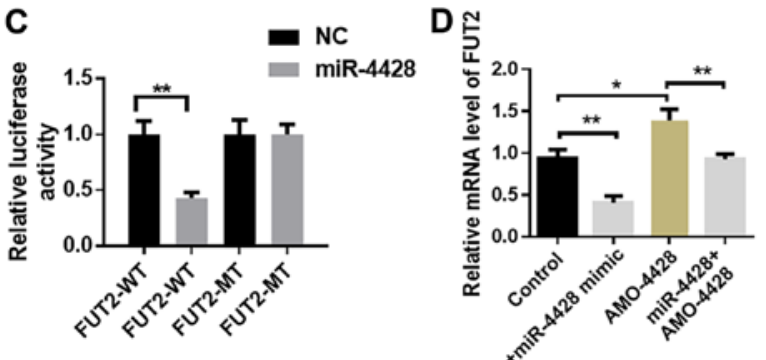

B

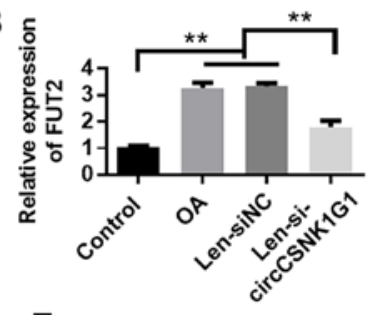

E

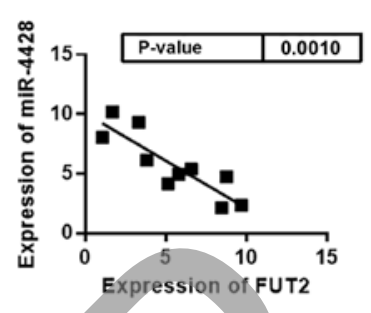

Figure 5. miR-4428 targets FUT2 in OA. (A) Predicted binding sites of miR-4428 and FUT2 mRNA. (B) FUT2 expression following circCSNK1G1 knockdown in the rat model of OA. (C) Detection of luciferase activity to confirm the association between miR-4428 and FUT2. (D) Expression of FUT2 in cultured chondrocytes. ${ }^{*} \mathrm{P}<0.05,{ }^{* *} \mathrm{P}<0.01$. (E) Correlation analysis between miR-4428 and FUT2 mRNA expression in patients with OA ( $\left.\mathrm{n}=10, \mathrm{r}=-0.7597\right)$. OA, osteoarthritis; FUT2, fucosyltransferase 2.

A

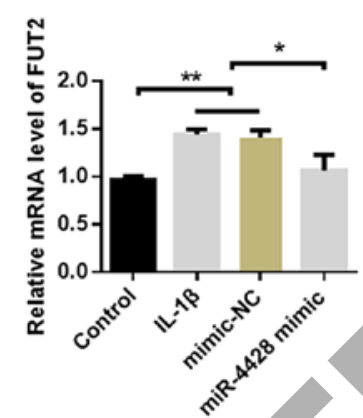

D
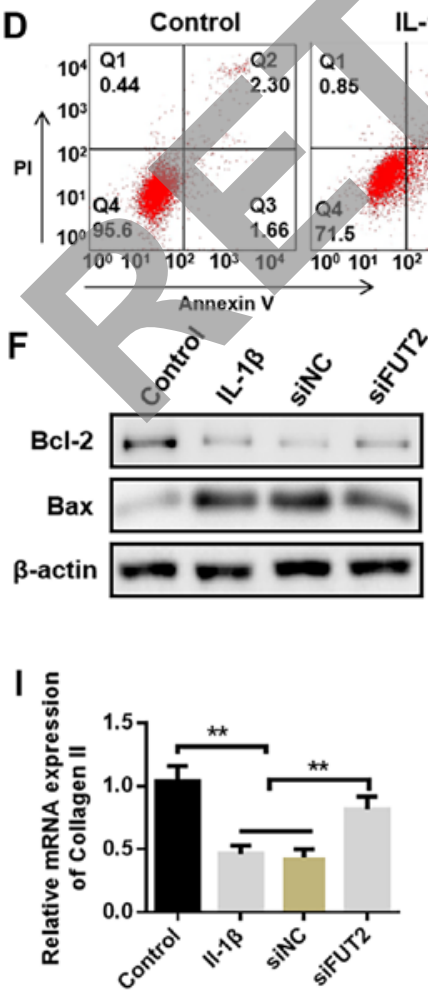

B

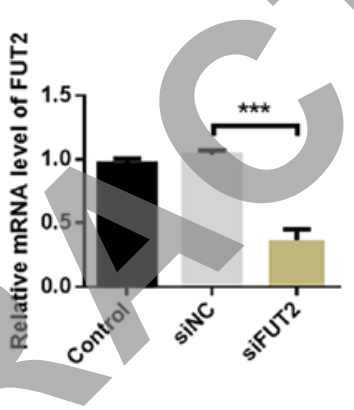

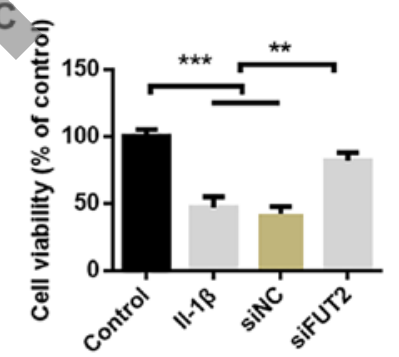

E

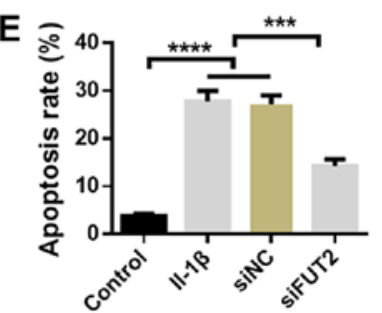

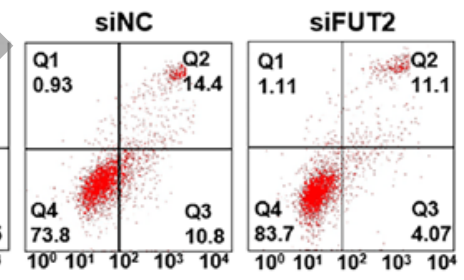
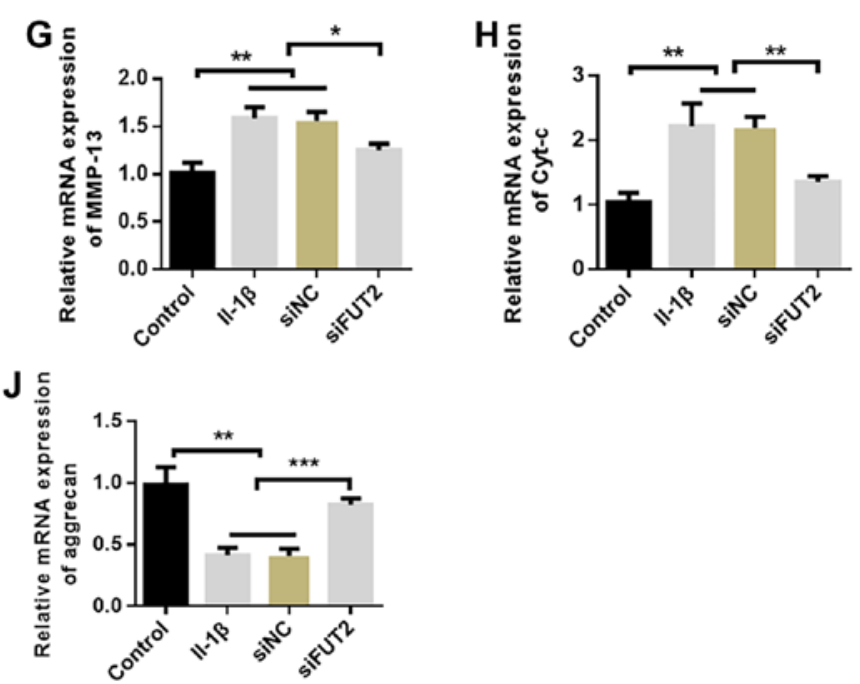

Figure 6. miR-4428 inhibits chondrocyte apoptosis by targeting FUT2. (A) Expression of FUT2 in cultured chondrocytes. (B) Expression of FUT2 following FUT2 siRNA knockdown. (C) Proliferation assays of cultured chondrocytes. (D and E) Percentage of apoptotic cells detected by flow cytometry assay. (F-J) Expression of Bax, Bcl-2, Cyt-c, MMP-13, collagen II and aggrecan in cultured chondrocytes was detected by western blot analysis or RT-qPCR "P<0.05, $\left.{ }^{* *} \mathrm{P}<0.01,{ }^{* * * *} \mathrm{P}<0.001,{ }^{* * * * *} \mathrm{P}<0.0001\right)$. OA, osteoarthritis; Cyt-c, cytochrome $c$; MMP-13, matrix metalloproteinase 13. 
arthritis. The aim of the present study was to examine the regulatory role of the circCSNK1G1/miR-4428/FUT2 axis in arthritis and to determine the cellular/molecular mechanisms of this axis.

Studies have demonstrated that some circRNAs are involved in the progression of arthritis. circRNA-CDRlas has been shown to be upregulated in OA tissue and targets miR-641 to increase MMP-13 and IL-6 expression (33). circ_0136474 has been shown to sponge miR-127-5p to upregulate MMP-13 expression and inhibit chondrocyte proliferation (34). circRNAs, such as hsa_circRNA_0020014 and hsa_circ_0032131, can serve as diagnostic biomarkers and treatment targets for OA (35-37). The present study provided some novel findings. First, circCSNK1G1 expression negatively correlated with miR-4428 expression; specifically, the former was upregulated, whereas the latter was downregulated in OA. Second, the role of circCSNK1G1 in OA was confirmed and it was found that the overexpression of circCSNK1G1 promoted the progression of OA. circCSNK1G1 was upregulated in OA-affected cartilage and induced chondrocyte apoptosis and EMC degradation by sponging the target of miR-4428. miR-4428 conferred protective effects against OA by inhibiting FUT2 expression. FUT2 has been shown to trigger airway inflammation via C3a production and dendritic cell accumulation (38); it has also been shown to promote breast cancer development by regulating cell proliferation, adhesion and migration (39). FUT2 is upregulated in OA-affected tissues, and miR-17-5p targets FUT2 to regulate the $\mathrm{Wnt} / \beta$-catenin pathway and inhibit OA development $(18,40,41)$. In the present study, FUT2 and circCSNK1G1 were similarly upregulated in OA-affected cartilage. The inhibition of FUT2 expression resulted in beneficial effects against OA development. As a target gene of miR-4428, FUT2 may mediate the role of the circCSNK1G1/miR-4428 axis in promoting OA. However, the pathways downstream of FUT2 were not detected in the present study.

Chondrocytes are the only cells that make up articular cartilage. The balance between chondrocyte apoptosis and proliferation plays a pivotal role in maintaining chondrocyte cell numbers in cartilage. Apoptosis refers to the programmed death of cells triggered by caspases (42). There is evidence to indicate that apoptosis plays a pivotal role in the onset and development of OA (43). The H19/miR-675/COL2A1 axis is activated and induces apoptosis in OA (44). The lncRNA-CIR/miR-27/MMP13 axis regulates chondrocyte apoptosis in OA (45). The DLL1/PI3K/AKT (46), MyD88/NF-kB/p38MAPK (47) and BCL-2/MAPK/JNK (48) pathways have also been reported to regulate chondrocyte apoptosis in OA. Thus, the chondrocyte cell number is the foundation of ECM formation and degradation (49).

The present study revealed new aspects of the cellular function and pathophysiological role of circCSNK1G1 and miR-4428, both of which may be considered potential molecular targets for the treatment of OA. Despite its numerous benefits, the present study also includes some shortcomings. For example, whether other miRNAs are sponged by circCSNK1G1 in OA remains unknown. Thus, future studies are required to focus on clarifying the underlying pathways of circCSNK1G1.
In conclusion, the present study, to the best of our knowledge, is the first to report the role of the circCSNK1G1/miR-4428/FUT2 axis in OA development via the regulation of chondrocyte apoptosis and ECM degradation. The results reveal that the circCSNK1G1/miR-4428/FUT2 axis may present a novel therapeutic target for the treatment of OA.

\section{Acknowledgements}

Not applicable.

\section{Funding}

The present study was funded by the Sanming Project of Medicine in Shenzhen (grant no. SZSM201602087); and the Shenzhen Futian District Health and Public Welfare Research Project (grant no. FTWS2018066)

\section{Availability of data and materials}

The datasets used and/or analyzed in the current study are available from the corresponding author on reasonable request.

\section{Authors' contributions}

ZY participated in the design of the study. JX analyzed and interpreted the data. JX, RW and WZ performed the experiments and collected important background information. XC performed the literature search, data acquisition and manuscript editing. All authors read and approved the final manuscript.

\section{Ethics approval and consent to participate}

All patients were fully informed of the experimental aims and procedures and provided written informed consent. The Ethics Committee of Shenzhen Futian Hospital for Rheumatic Disease approved the study. All animal procedures were approved by the Ethics Committee of Shenzhen Futian Hospital for Rheumatic Disease.

\section{Patient consent for publication}

Not applicable.

\section{Competing interests}

The authors declare that they have no competing interests.

\section{References}

1. Cross M, Smith E, Hoy D, Nolte S, Ackerman I, Fransen M, Bridgett L, Williams S, Guillemin F, Hill CL, et al: The global burden of hip and knee osteoarthritis: estimates from the global burden of disease 2010 study. Ann Rheum Dis 73: 1323-1330, 2014.

2. Loeser RF, Goldring SR, Scanzello CR and Goldring MB: Osteoarthritis: A disease of the joint as an organ. Arthritis Rheum 64: 1697-1707, 2012.

3. Memczak S, Jens M, Elefsinioti A, Torti F, Krueger J, Rybak A, Maier L, Mackowiak SD, Gregersen LH, Munschauer M, et al: Circular RNAs are a large class of animal RNAs with regulatory potency. Nature 495: 333-338, 2013. 
4. Szabo L and Salzman J: Detecting circular RNAs: Bioinformatic and experimental challenges. Nat Rev Genet 17: 679-692, 2016.

5. Cocquerelle C, Mascrez B, Hétuin D and Bailleul B: Mis-splicing yields circular RNA molecules. FASEB J 7: 155-160, 1993.

6. Liu Q, Zhang X, Hu X, Dai L, Fu X, Zhang J and Ao Y: Circular RNA related to the chondrocyte ECM Regulates MMP13 expression by functioning as a MiR-136 'sponge' in human cartilage degradation. Sci Rep 6: 22572, 2016.

7. Xu H, Guo S, Li W and Yu P: The circular RNA Cdrlas, via miR-7 and its targets, regulates insulin transcription and secretion in islet cells. Sci Rep 5: 12453, 2015.

8. Wang K, Long B, Liu F, Wang JX, Liu CY, Zhao B, Zhou LY, Sun T, Wang M, Yu T, et al: A circular RNA protects the heart from pathological hypertrophy and heart failure by targeting miR-223. Eur Heart J 37: 2602-2611, 2016.

9. Li J, Yang J, Zhou P, Le Y, Zhou C, Wang S, Xu D, Lin HK and Gong Z: Circular RNAs in cancer: Novel insights into origins, properties, functions and implications. Am J Cancer Res 5: $472-480,2015$

10. Hansen TB, Jensen TI, Clausen BH, Bramsen JB, Finsen B, Damgaard CK and Kjems J: Natural RNA circles function as efficient microRNA sponges. Nature 495: 384-388, 2013.

11. Ji Y, Fang QY, Wang SN, Zhang ZW, Hou ZJ, Li JN and Fu SQ Lnc-RNA BLACAT1 regulates differentiation of bone marrow stromal stem cells by targeting miR-142-5p in osteoarthritis. Eur Rev Med Pharmacol Sci 24: 2893-2901, 2020.

12. Liu C, Gao J, Su G, Xiang Y and Wan L: MicroRNA-1202 plays a vital role in osteoarthritis via KCNQ1OT1 has-miR-1202-ETS1 regulatory pathway. J Orthop Surg Res 15: 130, 2020.

13. Ren T, Wei P, Song Q, Ye Z, Wang Y and Huang L: MiR-140-3p ameliorates the progression of osteoarthritis via targeting CXCR4. Biol Pharm Bull 43: 810-816, 2020.

14. Rademacher TW, Parekh RB and Dwek RA: Glycobiology. Ann Rev Biochem 57: 785-838, 1988.

15. Mizuochi T, Taniguchi T, Shimizu A and Kobata A: Structural and numerical variations of the carbohydrate moiety of immunoglobulin G. J Immunol 129: 2016-2020, 1982.

16. Lopman BA, Trivedi T, Vicuña Y, Costantini V, Collins N, Gregoricus N, Parashar U, Sandoval C, Broncano N, Vaca $\mathrm{M}$, et al: Norovirus infection and disease in an ecuadorian birth cohort: Association of certain norovirus genotypes with host FUT2 Secretor Status. J Infect Dis 211: 1813-1821, 2015

17. Chandler KB, Alamoud KA, Stahl VL, Nguyen BC, Kartha VK, Bais MV, Nomoto K, Owa T, Monti S, Kukuruzinska MA and Costello CE: $\beta$-Catenin/CBP inhibition alters epidermal growth factor receptor fucosylation status in oral squamous cell carcinoma. Mol Omics 16: 195-209, 2020.

18. Hu J, Wang Z, Pan Y, Ma J, Miao X, Qi X, Zhou H and Jia L: MiR-26a and miR-26b mediate osteoarthritis progression by targeting FUT4 via NF- $\mathrm{B}$ signaling pathway. Int J Biochem Cel Biol 94: 79-88, 2018

19. Huang M, He YR, Liang LC, Huang Q and Zhu ZQ: Circular RNA hsa circ 0000745 may serve as a diagnostic marker for gastric cancer. World J Gastroenterol 23: 6330-6338, 2017.

20. Charlier E, Relic B, Deroyer C, Malaise O, Neuville S, Collée J, Malaise MG and De Seny D: Insights on molecular mechanisms of chondrocytes death in osteoarthritis. Int J Mol Sci 17: 2146, 2016.

21. Mobasheri A: The future of osteoarthritis therapeutics: Emerging biological therapy. Curr Rheumatol Rep 15: 385, 2013.

22. Dequeker J and Luyten FP: The history of osteoarthritis-osteoarthrosis. Ann Rheum Dis 67: 5-10, 2008.

23. Ding C, Jones G, Wluka AE and Cicuttini F: What can we learn about osteoarthritis by studying a healthy person against a person with early onset of disease? Curr Opin Rheumatol 22: 520-527, 2010.

24. Goldring MB and Goldring SR: Articular cartilage and subchondral bone in the pathogenesis of osteoarthritis. Ann NY Acad Sci 1192: 230-237, 2010.

25. Pollard TC, Gwilym SE and Carr AJ: The assessment of early osteoarthritis. J Bone Joint Surg Br 90: 411-421, 2008

26. D'Adamo S, Cetrullo S, Minguzzi M, Silvestri Y, Borzì RM and Flamigni F: MicroRNAs and autophagy: Fine players in the control of chondrocyte homeostatic activities in osteoarthritis. Oxid Med Cell Longev 2017: 3720128, 2017.

27. Bartel DP: MicroRNAs: Genomics, biogenesis, mechanism, and function. Cell 116: 281-297, 2004

28. Malemud CJ: MicroRNAs and osteoarthritis. Cells 7: 92, 2018.

29. He J, Zhang J and Wang D: Down-regulation of microRNA-216b inhibits IL-1 $\beta$-induced chondrocyte injury by up-regulation of Smad3. Biosci Rep 37: BSR20160588, 2017.
30. Lin Z, Tian XY, Huang XX, He LL and Xu F: microRNA-186 inhibition of PI3K-AKT pathway via SPP1 inhibits chondrocyte apoptosis in mice with osteoarthritis. J Cell Physiol 234: 6042-6053, 2019.

31. Ma Y, Wu Y, Chen J, Huang K, Ji B, Chen Z, Wang Q, Ma J, Shen $S$ and Zhang J: miR-10a-5p promotes chondrocyte apoptosis in osteoarthritis by targeting HOXA1. Mol Ther Nucleic Acids 14: 398-409, 2019.

32. Li H, Yang HH, Sun ZG, Tang HB and Min JK Whole-transcriptome sequencing of knee joint cartilage from osteoarthritis patients. Bone Joint Res 8: 288-301, 2019.

33. Zhang W, Zhang C, Hu C, Luo C, Zhong B and Yu X: Circular RNA-CDRlas acts as the sponge of microRNA-641 to promote osteoarthritis progression. J Inflamm (Lond) 17: 8, 2020.

34. Li Z, Yuan B, Pei Z, Zhang K, Ding Z, Zhu S, Wang Y, Guan Z and Cao Y: Circ 0136474 and MMP-13 suppressed cell proliferation by competitive binding to miR-127-5p in osteoarthritis. J Cell Mol Med 23: 6554-6564, 2019.

35. Wang Y, Wu C, Yang Y, Ren Z, Lammi MJ and Guo X: Preliminary exploration of hsa_circ_0032131 levels in peripheral blood as a potential diagnostic biomarker of osteoarthritis. Genet Test Mol Biomarkers 23: 717-721, 2019.

36. Wang Y, Wu C, Zhang Y, Yang Y, Ren Z, Lammi MJ and Guo X: Screening for differentially expressed circRNA between Kashin-Beck disease and osteoarthritis patients based on circRNA chips. Clin Chim Acta 501: 92-101, 2020.

37. Li HZ, Lin Z, Xu XH, Lin N and Lu HD: The potential roles of circRNAs in osteoarthritis: A coming journey to find a treasure. Biosci Rep 38: BSR20180542, 2018

38. Saku A, Hirose K, Ito T, Iwata A, Sato T, Kaji H, Tamachi T, Suto A, Goto Y, Domino SE, et al: Fucosyltransferase 2 induces lung epithelial fucosylation and exacerbates house dust mite-induced airway inflammation. J Allergy Clin Immunol 144: 698-709.e9, 2019

9. Lai TY, Chen IJ, Lin RJ, Liao GS, Yeo HL, Ho CL, Wu JC, Chang NC, Lee AC and Yu AL: Fucosyltransferase 1 and 2 play pivotal roles in breast cancer cells. Cell Death Discov 5: 74, 2019. 0. Dou P, Hu R, Zhu W, Tang Q, Li D, Li H and Wang W: Long non-coding RNA HOTAIR promotes expression of ADAMTS-5 in human osteoarthritic articular chondrocytes. Pharmazie 72: 113-117, 2017

41. Hu J, Wang Z, Shan Y, Pan Y, Ma J and Jia L: Long non-coding RNA HOTAIR promotes osteoarthritis progression via miR-17-5p/FUT2/ $\beta$-catenin axis. Cell Death Dis 9: 711, 2018.

42. D'Arcy MS: Cell death: A review of the major forms of apoptosis, necrosis and autophagy. Cell Biol Int 43: 582-592, 2019.

43. Musumeci G, Castrogiovanni P, Trovato FM, Weinberg AM, Al-Wasiyah MK, Alqahtani MH and Mobasheri A: Biomarkers of chondrocyte apoptosis and autophagy in osteoarthritis. Int J Mol Sci 16: 20560-20575, 2015.

44. Steck E, Boeuf S, Gabler J, Werth N, Schnatzer P, Diederichs S and Richter W: Regulation of H19 and its encoded microRNA-675 in osteoarthritis and under anabolic and catabolic in vitro conditions. J Mol Med (Berl) 90: 1185-1195, 2012.

45. Li YF, Li SH, Liu Y and Luo YT: Long noncoding RNA CIR promotes chondrocyte extracellular matrix degradation in osteoarthritis by acting as a sponge for Mir-27b. Cell Physiol Biochem 43: 602-610, 2017.

46. Zhang W, Hsu P, Zhong B, Guo S, Zhang C, Wang Y, Luo C, Zhan Y and Zhang C: MiR-34a enhances chondrocyte apoptosis, senescence and facilitates development of osteoarthritis by targeting DLL1 and regulating PI3K/AKT pathway. Cell Physiol Biochem 48: 1304-1316, 2018.

47. Ren Cand Liang Z: Piperine alleviates lipopolysaccharide-induced inflammatory injury by down-regulating microRNA-127 in murine chondrogenic ATDC5 cells. Biomed Pharmacother 103: 947-954, 2018.

48. Yu CD, Miao WH, Zhang YY, Zou MJ and Yan XF: Inhibition of miR-126 protects chondrocytes from IL-1 $\beta$ induced inflammation via upregulation of Bcl-2. Bone Joint Res 7: 414-421, 2018.

49. Sandell LJ and Aigner T: Articular cartilage and changes in arthritis. An introduction: Cell biology of osteoarthritis. Arthritis Res 3: 107-113, 2001.

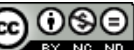

This work is licensed under a Creative Commons Attribution-NonCommercial-NoDerivatives 4.0 International (CC BY-NC-ND 4.0) License. 